\title{
Edelweiss à la recherche de la matière noire
}

\author{
Martine Stern, pour la collaboration Edelweiss $\left(^{*}\right)$ \\ Institut de physique nucléaire de Lyon \\ UCBL - IN2P3-CNRS - 4, rue Enrico Fermi - 69622 Villeurbanne Cedex - France
}

\begin{abstract}
À la frontière entre cosmologie et physique des particules, la recherche de la matière noire de l'Univers sous forme de particules stables mais non encore observées, les "wimps", se poursuit depuis une vingtaine d'années. Quelques expériences ont atteint récemment une sensibilité suffisante pour mettre à l'épreuve les modèles de physique des particules où ces wimps trouvent leur place dans un cadre théorique cohérent. Edelweiss, un dispositif situé au laboratoire souterrain de Modane, est l'une de ces expériences pionnières.
\end{abstract}

L'hypothèse de la matière noire naît en 1933. Cette annéelà, F. Zwicky étudie la dispersion des vitesses de galaxies dans l'amas de Coma. II remarque que, pour que celles-ci soient liées gravitationnellement, il faut supposer que leur masse est cent fois supérieure à ce que l'on estime à partir de leur luminosité. Cependant, cette idée d'une matière sombre reste dans l'oubli, voire le déni, une quarantaine d'années durant ; la question rebondit dans les années 60-70 avec notamment les observations de V. Rubin sur les spectres lumineux des galaxies spirales. Depuis, les physiciens et les astronomes n'ont de cesse de vouloir résoudre l'énigme de cette « masse manquante».

\section{La matière noire}

Deux types d'arguments sont avancés pour justifier l'existence de cette matière noire. Le premier type est lié aux résultats des observations des astrophysiciens sur la dynamique des objets célestes. Les étoiles des parties externes des galaxies tournent beaucoup trop vite autour de leur centre commun, semblant contredire la loi de Newton dans l'hypothèse où la masse des galaxies est essentiellement due à ces mêmes étoiles. II existerait donc une composante sombre, non encore observée, sans quoi les étoiles du bord des galaxies s'en échapperaient et se disperseraient. (Le même argument s'applique aux galaxies au sein des amas de galaxies.)

La problématique de la masse manquante est également justifiée par un second type d'argument, d'ordre cosmologique. Le modèle cosmologique standard du Big Bang s'appuie sur des observations robustes (rayonnement cosmologique à 2,7 K, abondance des éléments légers, structuration de la matière à grande échelle, luminosité apparente des supernovae lointaines) [1]. Les principales conclusions que l'on tire aujourd'hui de ces observations sont les suivantes : la densité totale de l'Univers est très voisine de la densité critique, $\rho_{c}=10^{-29} \mathrm{~g} / \mathrm{cm}^{3}$; l'ensemble de la matière, rassemblée dans les amas de galaxies, représente environ $30 \%$ de cette densité totale ${ }^{(1)}$; la matière ordinaire (les atomes) n'est pas assez abondante pour rendre compte de toute la masse des amas - il s'en faut d'un facteur 6 ; enfin, les étoiles ellesmêmes ne constituent que $10 \%$ de la matière ordinaire (soit environ $0,5 \%$ de la densité totale de l'Univers, ou 0,03 protons par mètre cube en moyenne).

Les observations cosmologiques ne sont donc cohérentes que si la quasi-totalité de la matière dans l'Univers est sous une forme non lumineuse, dont la grande majorité n'est même pas constituée d'atomes. Cette matière ordinaire est appelée baryonique, car les protons et les neutrons sont membres de la famille des baryons ${ }^{(2)}$; par opposition, la composante majo-

1. Les $70 \%$ restants sont sous forme d'« énergie noire ", une nouvelle composante de la matière, répartie uniformément dans l'Univers, et dont la densité reste constante, indépendamment de l'expansion. Cette énergie noire serait responsable de l'accélération de l'expansion, découverte récemment. ritaire de la matière reçoit l'appellation de non-baryonique. Se pose alors naturellement la question de la nature de cette composante, invisible bien que dominante au sein des galaxies.

Une des premières idées fut de rechercher cette matière sous forme de particules connues, les neutrinos. Les progrès récents montrent que cet espoir était illusoire, car leur masse ne semble pas assez élevée [2]. De plus les neutrinos ne peuvent rendre compte quantitativement de la structuration de la matière en amas de galaxies. D'autres candidats ont été proposés par les théoriciens. Par exemple, le respect de la symétrie CP par les interactions fortes ${ }^{(3)}$ a conduit des théoriciens à postuler l'existence de l'" axion ", particule de masse très faible $\left(\sim \mu \mathrm{eV}, 10^{-12}\right.$ fois celle de l'électron), que l'on n'a encore jamais observée.

Le candidat le plus populaire est en fait issu d'une hypothèse phénoménologique: constatant que les observations pouvaient s'exprimer en termes de particules massives, encore inconnues et interagissant très faiblement avec la matière, des théoriciens ont postulé dans les années 1980 l'existence des wimps (acronyme de Weakly Interacting Massive Particles, et qui signifie « mauviettes » en français) ${ }^{(4)}$. La recherche de ces wimps a débuté dès la fin des années 80 [3]. Par la suite, on a proposé que les wimps soient les " neutralinos », particules dont l'existence est prédite par des théories de supersymétrie [4], qui généralisent et englobent le " modèle standard » de la physique des particules. L'expérience Edelweiss - Expérience pour détecter les wimps en site souterrain - est une collaboration européenne de 9 laboratoires (dont la liste est donnée en encadré p. 20) ayant pour objectif de les détecter.

\section{Phénoménologie du wimp}

La manière dont les galaxies et les amas de galaxies sont aujourd'hui répartis impose aux particules recherchées d'être assez lourdes et « froides »; on entend par là non relativistes durant le Big Bang (par contraste, les neutrinos sont légers et " chauds"). Ces particules non baryoniques seraient donc des vestiges du Big Bang qui, à cause de leurs faibles interactions et de leur grande masse, se sont essentiellement concentrés par gravitation au cours de l'expansion de l'Univers. Elles ont, du même coup, entraîné dans leur sillage la matière baryonique ordinaire, donnant ainsi naissance aux amas de galaxies.

Cette capacité de servir de "germe » aux amas contraint la valeur de leur masse et de l'intensité de leurs interactions, sans les déterminer précisément : ces deux quantités couvrent un vaste domaine, qui coïncide assez bien avec les exi-

2. Les baryons sont des particules composées de trois quarks.

3. La symétrie CP échange particules et antiparticules, et inverse simultanément les trois coordonnées d'espace.

4. Les wimps s'opposaient alors aux machos (pour MAssive Compact Halo Object) baryoniques, d'hypothétiques astres sombres des halos galactiques, dont l'existence n'a pas été confirmée par les programmes de recherche. 
gences des modèles supersymétriques [4]. Basés sur une nouvelle symétrie entre bosons et fermions, ces modèles prédisent l'existence de nombreuses nouvelles particules. Comme aucune d'entre elles n'a été observée à ce jour, leurs masses doivent être élevées (à moins que les modèles ne soient pas pertinents). La plus légère de ces nouvelles particules serait un fermion stable, le neutralino. Électriquement neutre, elle est sa propre anti-particule ${ }^{(5)}$. Elle serait une superposition quantique des partenaires supersymétriques de bosons neutres - le photon et le Z, ainsi que le boson de Higgs. La recherche du neutralino auprès de l'accélérateur Lep (Large Electron Positron collider) du Cern a été infructueuse, ce qui impose que sa masse soit supérieure à $45 \mathrm{GeV}$, soit environ 50 fois la masse du proton [5].

Des simulations numériques indiquent qu'au niveau de notre galaxie, les wimps sont supposés former un halo à peu près sphérique, sans mouvement de rotation d'ensemble. Leurs vitesses au sein de ce volume, beaucoup plus grand que celui de la Galaxie visible (figure 1), sont comparables à celles des étoiles, mais avec une distribution maxwellienne, isotrope. Le Soleil, comme les autres étoiles du disque, est au contraire en rotation autour du centre de la Galaxie. Aussi, à notre échelle, nous serions «balayés " par un vent de wimps dont la densité locale est de $0,3 \mathrm{GeV} / \mathrm{cm}^{3}$. Comme la vitesse de la Terre par rapport au Soleil $(30 \mathrm{~km} / \mathrm{s})$ s'ajoute vectoriellement à celle du Soleil autour du centre de la galaxie (200 $\mathrm{km} / \mathrm{s}$ ), on prédit des variations annuelles du flux de wimps, d'environ $5 \%$.

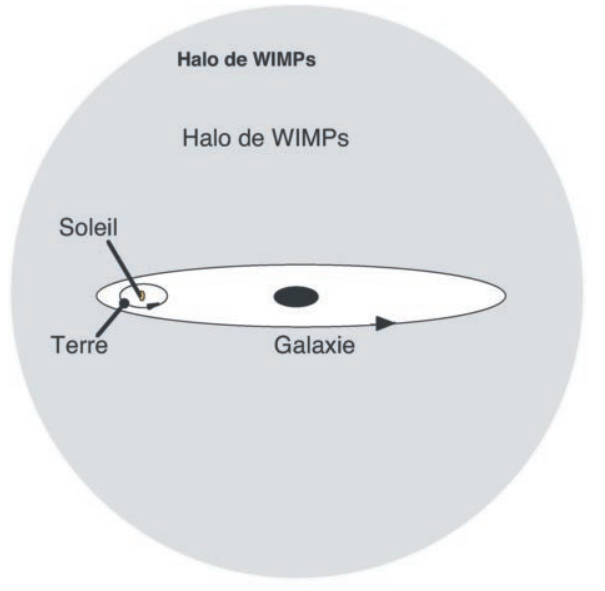

Figure 1 : Illustration du halo de wimps entourant notre Voie Lactée.

Il existe deux modes de détection des wimps galactiques. La méthode indirecte consiste à repérer une source cosmique émettant les produits d'annihilation de ces particules. La détection directe consiste à observer en laboratoire l'interaction du wimp avec un noyau ; elle devrait être sensible à la variation annuelle du flux de wimps. En outre, les futurs accélérateurs de haute énergie, tel le LHC du Cern, sont susceptibles de révéler la production de paires de neutralinos, à condition que leur masse soit inférieure à quelques centaines de GeV.

\section{Principe de la méthode de détection}

La collaboration Edelweiss vise la détection directe de collisions élastiques de wimps sur des noyaux. Dans la limite non relativiste, il n'existe que deux contributions à la diffusion élastique d'un neutralino sur un nucléon (neutron ou proton), dites scalaire et axiale, respectivement indépendante $(\mathrm{SI})$ et dépendante (SD) du spin du noyau. La section efficace d'une

5. Cela lui permet de s'annihiler par paire dans des lieux où il en existerait une densité suffisante (centre des galaxies, centre du Soleil, voire de la Terre). interaction de type SI est environ $\mathrm{A}^{2}$ fois plus grande que celle d'une interaction SD (A est le nombre de masse du noyau). La distribution des énergies de recul $E_{R}$ communiquées aux noyaux-cibles par les wimps est présentée sur la figure 2 (dans le cas SI) ; elle est exponentiellement décroissante, avec une pente reliée à la masse du wimp et un taux intégré proportionnel à la section efficace et au flux de wimps. On exprime usuellement ce taux en nombre de reculs par jour et par kilogramme de matériau cible.

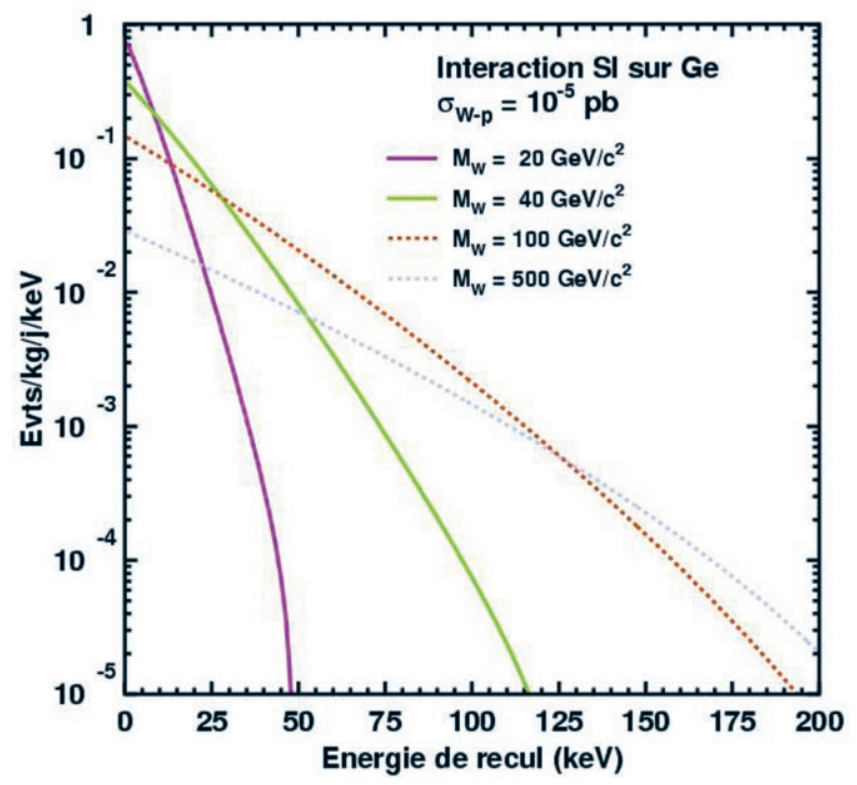

Figure 2 : Spectres théoriques des énergies de recul $E_{R}$ dans une cible de $G e$ pour des wimps de différentes masses, ayant une section efficace de diffusion sur un proton de $10^{-5} \mathrm{pb}$, et pour une densité locale de matière noire de $300000 \mathrm{GeV} / \mathrm{m}^{3}$

Comment identifier et mesurer ces reculs nucléaires dus à des interactions de wimps ? La quasi-totalité de l'énergie déposée est transférée au milieu sous forme de phonons. Dès les années 80 , le bolomètre ou détecteur thermique a paru adapté à ce type de mesures du fait de son très bas seuil théorique de détection en énergie des phonons. Un tel détecteur est constitué d'un matériau absorbeur - où la particule dépose l'énergie $E_{R}$ - et d'un thermomètre collé sur l'absorbeur. L'élévation de température mesurée est d'autant plus grande que la capacité calorifique de l'absorbeur est faible, d'où la nécessité de travailler à très basse température, dans un cryostat auquel le bolomètre est relié par une fuite thermique. Le cryostat à dilution d'Edelweiss, basé sur les propriétés du mélange ${ }^{3} \mathrm{He}-{ }^{4} \mathrm{He}$, permet d'atteindre $10 \mathrm{mK}$.

Les thermomètres ou senseurs adoptés dans la phase Edelweiss-1 sont en germanium dopé (leur taille est d'un $\mathrm{mm}^{3}$ ) ; ils fonctionnent à la transition métal-isolant grâce à un fort niveau de dopage en gallium, obtenu par transmutation nucléaire sous flux de neutrons thermiques. L'élévation de température induite par une collision entre wimp et noyau, de l'ordre du microkelvin, entraîne une variation de résistance aux bornes du senseur, lue comme une variation de tension dont l'optimisation dépend du courant injecté. La grande liberté dans le choix du matériau-cible, doublée de la simplicité du principe de détection, justifie l'appellation de détecteur « universel ».

Cependant l'existence d'un bruit de fond ressemblant aux chocs de wimps impose de disposer d'informations supplémentaires pour distinguer les wimps d'autres particules. II existe deux autres signaux susceptibles d'être produits dans le même matériau absorbeur et donc compatibles avec cette tech- 
nique du bolomètre : ils sont engendrés soit par l'excitation du milieu, soit par l'ionisation, tous deux sous l'effet du recul du noyau chargé. L'excitation du milieu produit de la lumière si le matériau est luminescent, alors que l'ionisation se traduit par la création de paires électron-trou si le matériau est semi-conducteur. Dans ce dernier cas, les paires électron-trou créées, mobiles, sont captées par des électrodes à la surface du cristal, et le courant résultant est mesuré. De la nature du matériau-cible choisi dépend donc le type de signal. Edelweiss a retenu l'ionisation. (La figure 3 illustre la formation des deux types de signaux, ionisation et chaleur, dans cette configuration.)

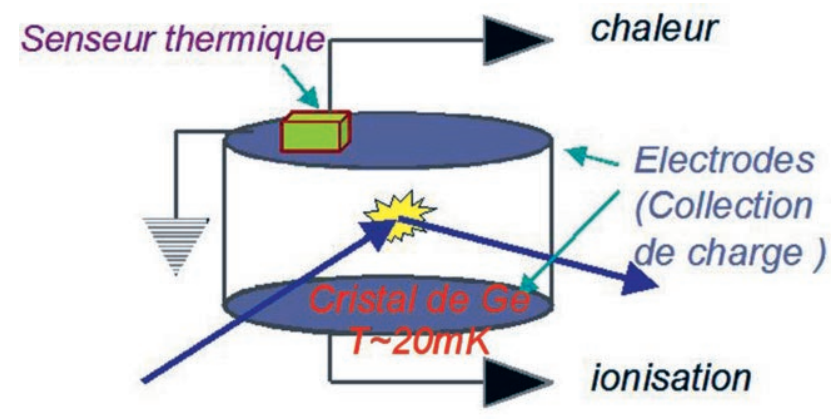

Figure 3 : Schéma de la formation et de la détection des deux signaux, ionisation et chaleur, sous l'effet du choc élastique d'un wimp sur un noyau du cristal de germanium.

La détection de l'ionisation limite le choix des cibles aux cristaux semi-conducteurs. La collaboration Edelweiss a adopté le germanium, pour lequel on atteint de grandes puretés (1 impureté pour $10^{11}$ atomes) et dont le nombre de masse élevé assure une plus grande section efficace que pour le silicium. Ce choix du germanium, constitué à $93 \%$ d'isotopes de spin nul, fait que l'expérience est surtout sensible à l'interaction de type scalaire. Le détecteur fonctionne en simple " chambre à ionisation », avec pour électrodes les surfaces du cristal rendues conductrices par un dépôt d'aluminium.

\section{La protection contre le bruit de fond}

\section{Le blindage d'Edelweiss-1}

Les wimps ne sont pas les seules particules susceptibles d'interagir dans le détecteur. II existe en effet un bruit de fond de neutrons, issus de la radioactivité environnante ou bien engendrés par les interactions de muons cosmiques autour du dispositif expérimental. Or la cinématique d'une collision élastique confère les mêmes énergies de recul aux noyaux de germanium lorsqu'ils sont frappés par des neutrons d'énergie cinétique de quelques $\mathrm{MeV}$ ou par des wimps de masse comprise entre 35 et $1000 \mathrm{GeV}$ ayant une vitesse de quelques centaines de $\mathrm{km} / \mathrm{s}$. On conçoit donc la nécessité de réduire le flux de neutrons (et de muons) dans l'environnement expérimental. Parallèlement, il faut aussi réduire le flux des photons $\mathrm{X}$ ou $\gamma$, eux aussi issus de la radioactivité ambiante ou des effets du rayonnement cosmique.

À la surface de la Terre, le rayonnement cosmique est considérable, de l'ordre de $10^{7}$ muons $/ \mathrm{m}^{2} /$ jour. Les taux d'interaction théoriques des wimps sont de plusieurs ordres de grandeur inférieurs à ceux engendrés par ce rayonnement cosmique : c'est pourquoi on est contraint d' « enterrer » le dispositif de détection d'une telle expérience sous une montagne ou dans une mine. La collaboration Edelweiss bénéficie du site du Laboratoire Souterrain de Modane (LSM, dans le tunnel routier du Fréjus), sous une couverture rocheuse de $1780 \mathrm{~m}$ qui divise par $210^{6}$ le flux de muons et arrête tous les autres rayons cosmiques. Le bruit de fond gamma à haute énergie est également réduit.

La radioactivité de la roche reste par contre inchangée. Elle est source d'un flux de neutrons, via des réactions de fission de l'uranium et du thorium présents dans la roche, ainsi que des réactions $(\alpha, n)$. Un triple blindage protège le dispositif expérimental de ces neutrons. À l'extérieur, $30 \mathrm{~cm}$ de paraffine réduisent d'un facteur 100 le flux de neutrons et dégradent considérablement leur énergie. Un second blindage de $15 \mathrm{~cm}$ de plomb réduit efficacement le flux de $\gamma$; il est suivi de $10 \mathrm{~cm}$ de cuivre pour arrêter les rayons $\mathrm{X}$ émis par le plomb et les photons de faible énergie. Cependant, le plomb est lui-même source de $\gamma$ de $45 \mathrm{keV}$ car il contient du ${ }^{210} \mathrm{~Pb}$, de durée de vie 22 ans. Un dernier écran est donc nécessaire au plus près des détecteurs : il est fait de plomb archéologique (appelé aussi « romain »), longtemps enfoui et donc exempt de ${ }^{210} \mathrm{~Pb}$. Le dispositif, qui comprend 3 détecteurs, est illustré sur la figure 4.

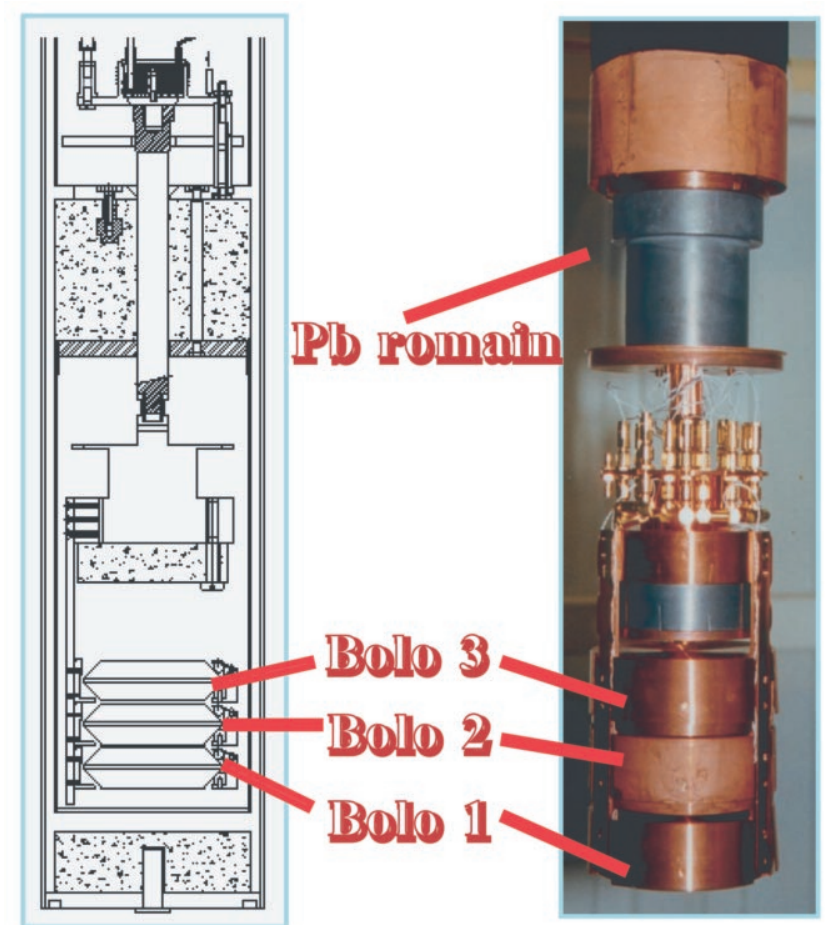

Figure 4 : Extrémité inférieure de l'unité de dilution à laquelle sont suspendus les trois détecteurs à l'intérieur d'un support de cuivre. Le plomb romain apparait en tacheté sur le schéma de gauche.

Un bruit de fond demeure dans l'atmosphère du laboratoire : il est lié au radon, seul élément gazeux dans la chaîne de désintégration de l'uranium. Son taux est maîtrisé par une circulation d'azote gazeux autour du cryostat contenant les détecteurs. Bien que tous les matériaux du dispositif expérimental aient été sélectionnés pour leur très basse radioactivité, une dernière source de bruit est la radioactivité intrinsèque du détecteur ainsi que celle du senseur. Faute de pouvoir la réduire, on la contrôle en étudiant une raie $d u{ }^{68} \mathrm{Ga}$ (produit lorsque des rayons cosmiques réagissent sur $d^{6}{ }^{68} \mathrm{Ge}$ ). Toutes ces précautions ont permis de réduire de façon drastique les taux d'événements de bruit de fond parasite observés depuis la mise en place de l'expérience.

\section{Discrimination des particules}

Malgré toutes ces précautions, il demeure un fond résiduel dû à la radioactivité naturelle et au rayonnement cosmique, qui donne lieu à des dépôts d'énergie dans le germanium. Comment discriminer alors les dépôts d'énergie induits par les wimps de ceux dus à ce fond résiduel ?

Tout d'abord, rappelons les différents types d'interaction mis en jeu. Les photons interagissent par effet photoélectrique, par diffusion Compton sur un électron de la cible ou par création de paires électron-positron. Ces trois processus sont causes de reculs électroniques. Les neutrons ambiants, 


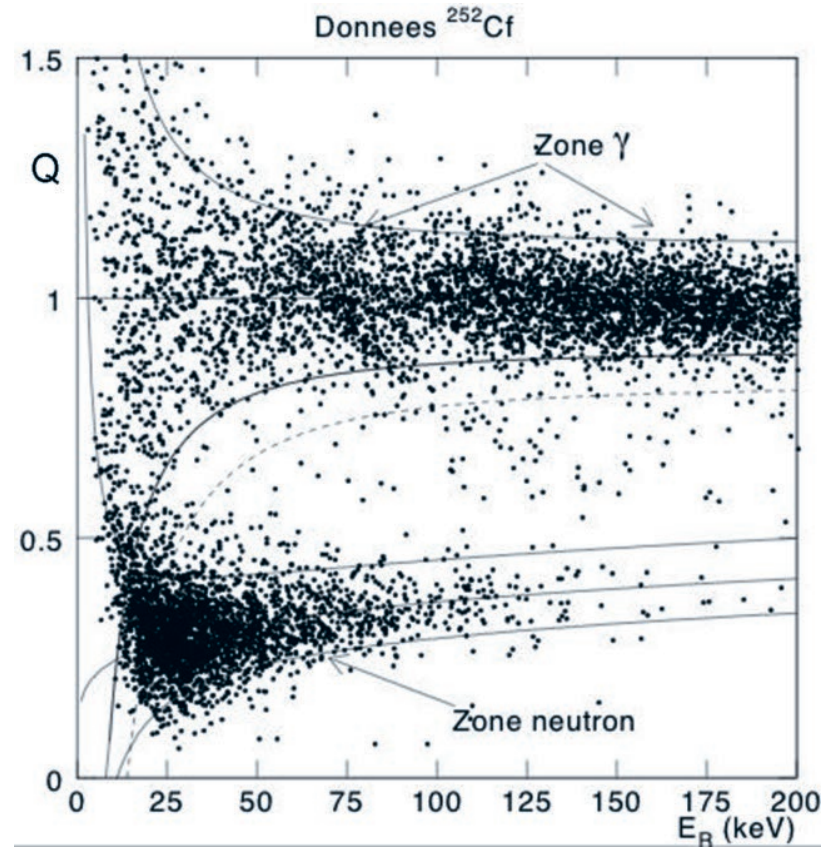

Figure 5 : Ce spectre biparamétrique $-Q$, facteur de quenching d'ionisation, en fonction de $E_{R}$ énergie de recul des électrons ou des ions - a été obtenu à l'aide d'une source radioactive de gammas et de neutrons. II montre nettement l'écart entre les deux réponses dans la cible de germanium. La dispersion des points suivant $Q$ est due à la résolution expérimentale, qui est moins bonne pour des reculs de plus faible énergie.

par contre, provoquent des reculs nucléaires par diffusion inélastique ou élastique, au même titre que les wimps. Les ions et les électrons (ou positrons) qui reculent dans le cristal perdent leur énergie en provoquant des ionisations successives le long de leur trajectoire. Mais, à énergie déposée identique, ces ions et électrons ont des pouvoirs d'ionisation très différents (à cause du grand écart entre leurs masses) ; la trajectoire d'un électron est beaucoup plus longue et erratique que celle d'un ion.

La grandeur physique qui traduit cette différence d'ionisation est le rapport du nombre de charges créées par chaque type de recul (ions ou électrons) au nombre moyen de charges créées par un recul électronique, pour la même énergie déposée ; on l'appelle facteur de quenching d'ionisation Q. Par définition, $Q$ est proche de 1 pour des reculs électroniques. Pour des reculs nucléaires, dans le cas du germanium, $Q$ est proche de 0,3 et ne varie que faiblement avec l'énergie de recul. Pour une même énergie $E_{R}$, la chaleur déposée dans les deux types de reculs est par contre la même : cela permet de discriminer entre recul nucléaire et électronique, en comparant $Q$ et l'énergie $E_{R}$, événement par événement (grâce au faible taux d'interactions dans le germanium, on mesure l'ionisation et la chaleur pour chaque événement séparément). La figure 5 en donne une illustration. Elle représente dans un plan $\left(E_{R}, Q\right)$ les données obtenues avec une source de ${ }^{252} \mathrm{Cf}$ émettant des $\gamma$ et des neutrons. On distingue nettement une « zone neutrons " (dite aussi zone de reculs nucléaires) autour de $Q$ $=0,3$ et une "zone $\gamma$ »(6) autour de $Q=1$. Pour s'affranchir du léger recouvrement entre les deux zones à basse énergie, on impose un seuil de détection sur $\mathrm{E}_{\mathrm{R}}$, de l'ordre de $25 \mathrm{keV}$.

L'espace entre les deux zones n'est néanmoins pas entièrement dépeuplé. On impute ces événements à des reculs d'électrons sur les bords externes du détecteur, où les inhomogénéités du champ électrique entraînent une collecte incomplète des charges. Afin de réduire le nombre de ces événements parasites, dits " de surface », les électrodes ont été segmentées en deux zones, centre et anneau de garde.

6. On parle de « zone gamma » par abus de langage ; ce sont en fait les électrons (et les positrons) produits par les interactions des photons qui sont détectés.
On ne retient que les événements où au moins $75 \%$ de la charge est collectée sur l'électrode centrale. La masse utile du détecteur s'en voit légèrement réduite.

\section{Les résultats d'Edelweiss-1}

Entre 2000 et 2003, plusieurs prises de données ont été effectuées avec 3 détecteurs cylindriques de $320 \mathrm{~g}(\mathrm{~h}=20 \mathrm{~mm}$, $\Phi=70 \mathrm{~mm}$, voir la figure 4). Dans une première phase, les données ont été accumulées en déclenchement sur la voie ionisation (c'est-à-dire en requérant un signal électrique supérieur à un certain seuil) avec deux bolomètres. Aucun événement susceptible d'être un recul nucléaire n'a été enregistré au dessus du seuil d'analyse $\left(E_{R}=20\right.$ et $30 \mathrm{keV}$ selon le détecteur); l'exposition totale était de 13,6 kg.j. Ce résultat se traduit par un diagramme d'exclusion dans le plan $\sigma$ (section efficace wimp-nucléon) versus $\mathrm{M}$ (masse du wimp). La limite correspondante est proche de celle représentée sur la figure 6 ; elle a permis à Edelweiss d'atteindre en 2002 la meilleure sensibilité au monde pour un wimp de masse supérieure à $30 \mathrm{GeV}$, soit $510^{-6} \mathrm{pb}[6]^{(7)}$. Elle correspond à un taux d'événement aussi bas que 0,2 événement $/ \mathrm{kg} / \mathrm{j}$.

Au cours de l'année 2003, deux prises de données ont été effectuées avec trois nouveaux détecteurs et deux types de déclenchements. Le fonctionnement simultané de ces trois détecteurs, occupant tout le volume utile du cryostat à dilution, constituait la dernière étape de la phase Edelweiss-1. Pour exemple, nous avons représenté sur la figure 7 la première série de données, effectuée avec un déclenchement sur la voie ionisation. On peut y observer au total 3 événements compatibles avec des reculs nucléaires (symboles violets), c'est-à-dire situés dans les zones wimps des 3 détecteurs (zones rouges)

La seconde série de données, non représentée ici, a été enregistrée avec un déclenchement sur la voie chaleur (c'est-

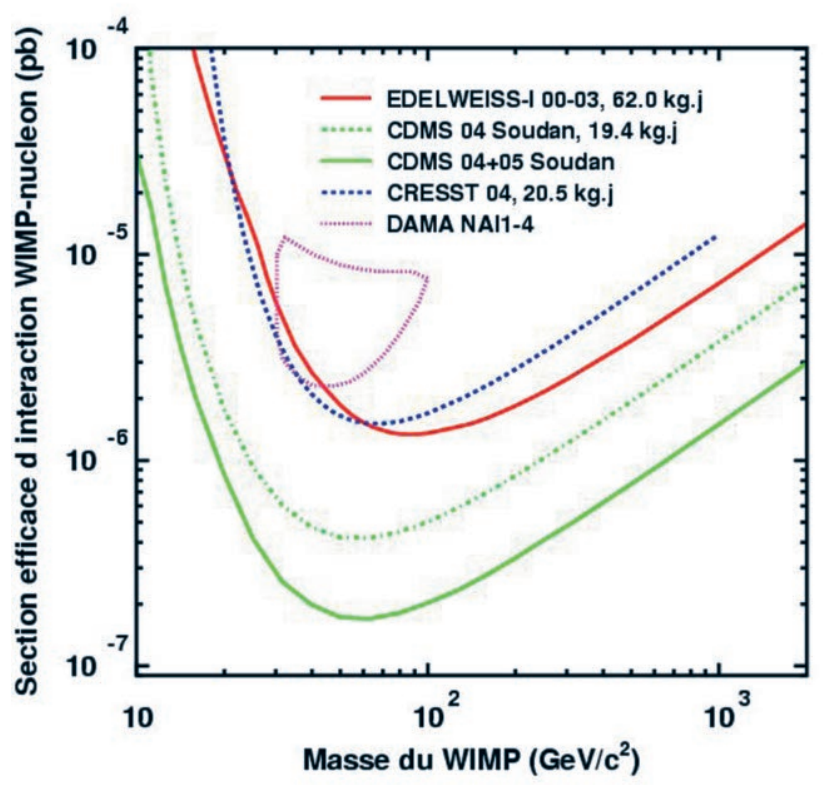

Figure 6 : Comparaison des contours d'exclusion calculés à 90\% de confiance des expériences Edelweiss [7] (courbe rouge), CDMS [8] (courbes vertes, en trait plein et tirets) et CRESST [9] (courbe bleue) avec le signal présenté par Dama [10] (à l'intérieur du contour violet). Les zones exclues sont celles situées au-dessus des contours, qui correspondent à des taux d'interaction plus élevés. Les diverses courbes sont calculées pour la même distribution de vitesse des wimps galactiques. Certaines prédictions de modèles supersymétriques [11,12], non représentées ici, couvrent en gros le domaine masse supérieure à $30 \mathrm{GeV}$ et section efficace plus petite que $10^{-5} \mathrm{pb}$.

7. 1 picobarn $=10^{-36} \mathrm{~cm}^{2}$. 

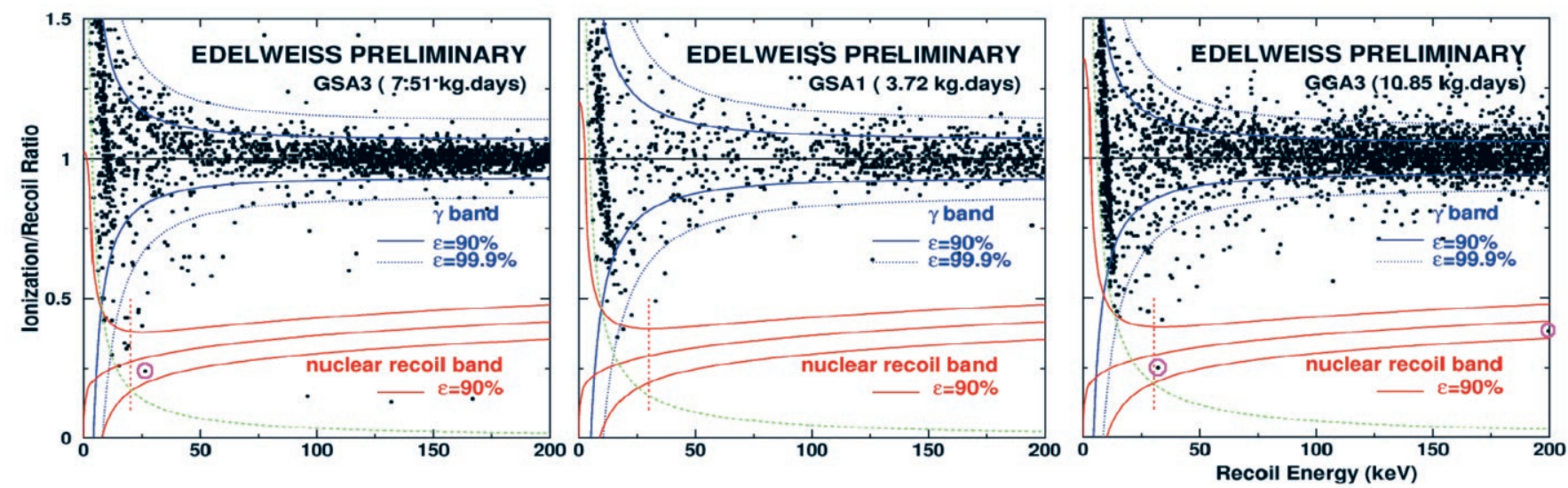

Figure 7 : Rapport du signal d'ionisation sur l'énergie de recul en fonction de l'énergie de recul, mesuré par l'expérience Edelweiss ; chaque graphe correspond à l'un des trois bolomètres pour les données de 2003. Noter les trois événements (symboles violets) à l'intérieur des zones "recul nucléaire " (en rouge), et audessus du seuil d'analyse $\left(E_{R}>20-30 \mathrm{keV}\right)$.

à-dire en exigeant un signal bolométrique au-delà d'un certain seuil). Ce type de déclenchement améliore l'efficacité à basse énergie grâce à une meilleure résolution et à l'absence de l'effet quenching sur la voie phonon. Dix-huit événements compatibles avec des reculs nucléaires ont été enregistrés audessus d'un seuil de $15 \mathrm{keV}$ dans cette dernière série.

Au total, dans l'ensemble des données d'Edelweiss-1 62 kg.j de données), il y a 40 événements compatibles avec des reculs nucléaires au-dessus de $15 \mathrm{keV}$. Une analyse globale combinant les divers lots de données vient d'être publiée [7]. La limite obtenue (figure 6) confirme le résultat de 2002. Sur cette même figure, on voit que le résultat d'Edelweiss- 1 est maintenant dépassé par celui de la collaboration américaine CDMS (Cold Dark Matter Search) [8] et ce, depuis le transfert de leur appareillage au fond de la mine de Soudan (Minnesota). L'expérience Dama (dans le tunnel du Gran Sasso, en Italie), qui a annoncé avoir observé un flux de wimps via sa variation annuelle (à l'intérieur du contour violet sur la figure 6) [10], voit maintenant ses résultats infirmés par trois expériences, Edelweiss, CDMS et CRESST (cette dernière, également dans le tunnel du Gran Sasso) [9] ; parmi ces expériences, seule Dama ne bénéficie pas de la double détection. Fait remarquable, les expériences atteignent aujourd'hui une sensibilité qui leur permet de rejeter, parmi les modèles supersymétriques $[11,12]$, ceux prédisant les sections efficaces les plus élevées $\left(10^{-5} \mathrm{pb}\right)$.

\section{Leçons d'Edelweiss-1}

La prise de données d'Edelweiss-1 s'est achevée en juillet 2003 après des développements sur les détecteurs, en vue de la phase Edelweiss-2. La sensibilité de l'expérience est à ce jour limitée par deux points. Le premier concerne les événements « de surface ": bien qu'identifiés entre les zones $\gamma$ et neutron, ils viennent sans doute aussi polluer la zone des reculs nucléaires. Le second est inhérent au principe de la détection, puisqu'il s'agit de la similitude des signaux de wimps et de neutrons.

Les signaux parasites " de surface » sont, pour la plupart, dus à des électrons de désintégration bêta proches des surfaces des électrodes. Ils sont victimes d'un déficit de collection des charges, d'où une valeur de $Q$ anormalement faible, ce qui les fait peupler l'espace entre zone gamma et zone wimps-neutrons sur la figure 5 . Ce processus peut être atténué par la pulvérisation de couches amorphes de quelques nanomètres d'épaisseur sur la surface du détecteur avant le dépôt d'aluminium. Un champ électrique plus élevé peut aussi réduire ce processus de diffusion.

Le second point concerne la discrimination entre neutron et wimp, théoriquement impossible à partir du seul quenching d'ionisation. Dans le cas des neutrons, on peut espérer observer des signaux en coïncidence entre détecteurs adjacents (ce qui est impossible pour les wimps, vu leur très faible probabilité d'interaction); cela fournit un moyen de rejeter les neutrons. Des simulations Monte-Carlo montrent néanmoins qu'il est probable que quelques-uns des 40 événements détectés soient dus à des neutrons, sans coïncidence. Le contrôle de ce bruit de fond neutronique apparaît donc capital pour la poursuite de l'expérience ; aussi le nouveau dispositif expérimental intègre-t-il dans sa conception des exigences plus sévères que dans la phase Edelweiss-1.

L'étape « $1 \mathrm{~kg}$ » (3 détecteurs de $320 \mathrm{~g}$ ) a permis de conclure que nous n'avons pas observé de wimps de masse supérieure à $30 \mathrm{GeV}$ qui aient une section efficace d'interaction, normalisée à celle du nucléon, supérieure à $510^{-6} \mathrm{pb}$. Afin de tester les prédictions d'une plus grande plage de modèles théoriques, notre prochain défi est de mesurer des sections efficaces 10 à 100 fois plus faibles. Ce défi est partagé par les deux équipes concurrentes, l'américaine CDMS et l'européenne CRESST, qui engagent simultanément une seconde phase (tout en conservant leur stratégie initiale d'un détecteur cryogénique + ionisation ou scintillation, respectivement).

\section{Perspectives}

L'expérience Edelweiss-1 est parvenue à une sensibilité à des taux d'interaction de l'ordre de 0,2 événement $/ \mathrm{kg} / \mathrm{j}$, sans toutefois détecter de wimps. Ces résultats sont demeurés les meilleurs au monde durant plus d'un an. L'amélioration de cette sensibilité passe par l'accroissement du nombre, et donc de la masse totale des détecteurs. Pour ce faire, l'expérience Edelweiss-2 vient de débuter en cette année 2006 au LSM. Elle dispose d'un nouveau cryostat, pouvant contenir une centaine de bolomètres de $320 \mathrm{~g}$, dont une dizaine est déjà installée. Ce nombre doit progressivement croître jusqu'à 30 (étape dite des « $10 \mathrm{~kg}$ ») ; sur une année, cela devrait nous rendre capables de mesurer des taux de 0,01 evt/kg/j.

La suite n'est pas entièrement planifiée, car il faut laisser place à des évolutions futures, voire à l'emploi de différents matériaux cibles. En particulier, différents procédés sont actuellement à l'étude pour obtenir une meilleure suppression des événements de surface, principale limitation du dispositif, soit en utilisant des électrodes de forme particulière, soit en analysant le déroulement temporel des signaux enregistrés.

Les expériences cryogéniques semblent en tout cas incontournables. Elles ont en effet le grand avantage de permettre un contrôle performant du bruit de fond, au contraire d'expériences sur cibles plus massives mais sans double détection. Cet avantage compense largement la complexité et les contraintes liées à la cryogénie, ainsi que la masse totale moindre. En ce qui 
concerne l'après Edelweiss-2, le passage à la trentaine de kilogrammes ne saurait lui-même combler totalement les attentes des physiciens. Pour tester au mieux l'hypothèse des wimps, une progression de plusieurs ordres de grandeur de la sensibilité est nécessaire ; elle imposera peut-être un rapprochement d'expériences actuellement concurrentes.

$\left(^{\star}\right)$ Les 9 laboratoires de la collaboration Edelweiss :

- Centre de Recherche sur les Très Basses Températures, CNRS, BP 166, 38042 Grenoble, France.

- Centre de Spectroscopie Nucléaire et de Spectrométrie de Masse, IN2P3-CNRS, Université Paris XI, bât 108, 91405 Orsay, France.

- Institut für Experimentelle Kernphysik, Universität Karlsruhe (TH), Gaedstrasse 1, 76128 Karlsruhe, Allemagne.

- Forchungszentrum Karlsruhe, Institut für Kernphysik, Postfach 3640, 76021 Karlsruhe, Allemagne.

- Institut de Physique Nucléaire de Lyon - UCBL, IN2P3-CNRS,

4, rue Enrico Fermi, 69622 Villeurbanne Cedex, France.

- CEA, Centre d'Études de Saclay, DSM/DRECAM, 91191 Gif-sur-Yvette Cedex, France

- CEA, Centre d'Études de Saclay, DSM/DAPNIA, 91191 Gif-sur-Yvette Cedex, France.

- Institut d'Astrophysique de Paris, INSU-CNRS, 98 bis, bd Arago, 75014 Paris, France.

- JINR Dubna, Joliot-Curie 6, 141980 Dubna, Russie.

\section{Remerciements}

L'auteur remercie Alain Milsztajn pour les nombreuses discussions scientifiques lors de la finalisation de cet article.

\section{Bibliographie}

[1] F. Combes, "Un nouvel Univers », Bull. SFP 144, 7 (2004)

[2] W.C. Haxton et B.R. Holstein, Am. J. Phys. 72, 18 (2004)

[3] S. Ahlen et al., Phys. Lett. B 195, 603 (1987)

[4] Pour une introduction aux théories de supersymétrie, se reporter au livre de G. Kane, Supersymétrie - Les lois ultimes de la matière dévoilées, Éditions Le Pommier (2003).

[5] J. Ellis et al., hep-ph/0004169 (2000)

[6] A. Benoît et al., Phys. Lett. B 545, 43 (2002)

[7] V. Sanglard et al., Phys. Rev. D71, 122002 (2005)

[8] D.S. Akerib et al., Phys. Rev. Lett. 93, 211301 (2004)

[9] G. Angloher et al., Astropart. Phys. 23, 325 (2005)

[10] R. Bernabei et al., Phys. Lett. B 480, 23 (2000)

[11] E.A. Baltz et P. Gondolo, Phys. Rev. D 67, 063503 (2003)

[12] A. Bottino et al., Phys. Rev. D 69, 037302 (2004) 